\title{
Two applications of time reversal mirrors: Seismic radio and seismic radar
}

\author{
Sherif M. Hanafy ${ }^{a)}$ and Gerard T. Schuster \\ King Abdullah University of Science and Technology (KAUST), Thuwal, Jeddah 23955, Saudi Arabia
}

(Received 13 February 2011; revised 8 July 2011; accepted 8 July 2011)

\begin{abstract}
Two seismic applications of time reversal mirrors (TRMs) are introduced and tested with field experiments. The first one is sending, receiving, and decoding coded messages similar to a radio except seismic waves are used. The second one is, similar to radar surveillance, detecting and tracking a moving object(s) in a remote area, including the determination of the objects speed of movement. Both applications require the prior recording of calibration Green's functions in the area of interest. This reference Green's function will be used as a codebook to decrypt the coded message in the first application and as a moving sensor for the second application. Field tests show that seismic radar can detect the moving coordinates $(x(t), y(t), z(t))$ of a person running through a calibration site. This information also allows for a calculation of his velocity as a function of location. Results with the seismic radio are successful in seismically detecting and decoding coded pulses produced by a hammer. Both seismic radio and radar are highly robust to signals in high noise environments due to the super-stacking property of TRMs. (C) 2011 Acoustical Society of America. [DOI: $10.1121 / 1.3621469$ ]
\end{abstract}

PACS number(s): 43.60.Dh, 43.20.El, 43.30.Cq, 43.50.Yw [RKS] Pages: 1985-1994

\section{INTRODUCTION}

The time-reversal method ${ }^{1,2}$ is an efficient approach for focusing pulsed waves through inhomogeneous media. In time-reversal acoustics a signal is recorded by an array of receivers, time-reversed and then re-transmitted into the medium. The re-transmitted signals propagate back through the same medium along both the direct waves and multiple scattering paths and refocus on the source. Prior to Fink's earliest papers, the TRM concept had been devised by ocean engineers for signal transmissions in the oceans. ${ }^{3}$ About the same time, exploration geophysicists were using a similar process known as migration, ${ }^{4}$ except they refocused recorded seismic reflections back to their place of origin. Indeed, if a numerical solution to the full wave equation was used they named this method reverse time migration. ${ }^{5-10}$

Two kinds of time-reversal are defined: the time reversal cavity (TRC) and the time reversal mirror (TRM). In the TRC the array of receivers completely surrounds the source and thus the time-reversed signals propagate backward through the medium and retrace their primary and multiple scattering paths traveled in the forward direction. In the TRM the array of receivers covers a limited area and just a smaller part of the field is captured.

Several publications describe lab and field TRM experiments such as using sonic waves in the ocean,,${ }^{3,11-14}$ using acoustic waves in a laboratory experiment, ${ }^{15-17}$ using EM waves ${ }^{18}$ and using seismic waves. ${ }^{19-21}$ The method used in Ref. 20 is very similar to what we will employ in this work. In that work a TRM is used to find the location and excitation time of unknown seismic sources in the subsurface. The method was tested on synthetic and field data. One of the

\footnotetext{
a) Author to whom correspondence should be addressed. Electronic mail: sherif.geo@gmail.com
}

applications they addressed was to locate trapped miners in collapsed mines. Results with both synthetic and field data showed that a TRM system can precisely locate trapped miners even with data having a 1:2000 signal-to-random-noise ratio, where white-noise was synthetically generated and added to the field data. ${ }^{20}$

In the current work we discuss two applications of seismic TRMs. First, we demonstrate how to, similar to a radio, receive and decipher coded messages from trapped miners, and second how to, similar to surveillance radar, track moving objects within an area of interest. TRM calibration records are required before receiving and decoding messages or tracking moving objects. These records will be used to decode the coded message or track moving objects, and should be recorded beforehand with a roughly 4:1 signal-to-noise ratio (SNR) or more so that signals can be recognized from noise.

This paper is organized into 5 parts: (1) the introduction, (2) the TRM theory section for both applications, (3) a description of the field test and results for the seismic radio application and (4) a description of the field test and results for tracking moving objects, and (5) the last part provides a discussion and summarizes the results.

\section{THEORY}

The TRM is similar to standard poststack migration except that trial time shifts are introduced into seismic records to compensate for the unknown source location and excitation time. ${ }^{20}$ The migration images (obtained by poststack migration of passive seismic data from a buried point source) are compared for different time shifts and the localized maxima of migration amplitudes pinpoints the unknown source locations. The migration image $m(\mathbf{x}, t)$ is given by ${ }^{1,17}$

$$
m(\mathbf{x}, t)=\sum_{g} d\left(\mathbf{g}, t \mid \mathbf{s}, t_{\text {source }}\right) * g(\mathbf{x},-t \mid \mathbf{g}, 0)
$$


where, for example, $g(\mathbf{x},-t \mid \mathbf{g}, 0)=A(\mathbf{g}, \mathbf{x}) \delta\left(t+\tau_{x g}\right)$ is the asymptotic acausal Green's function for an inhomogeneous medium with the geometrical spreading factor denoted by $A(\mathbf{g}, \mathbf{x}), d\left(\mathbf{g}, t \mid \mathbf{s}, t_{\text {source }}\right)$ represents the passive data recorded at time $t$ and at location $\mathbf{g}$ for a source at $\mathbf{S}$ with unknown excitation time $t_{\text {source }}$; here, the variable $t$ in $m(\mathbf{x}, t)$ is the trial time shift to compensate for a non-zero excitation time, and $\mathbf{x}$ is the trial image position. Choosing the trial time $t \rightarrow t_{\text {source }}$ and trial source location $\mathbf{x} \rightarrow \mathbf{s}$ yields the maximum magnitude of the migration image at the actual source location $\mathbf{s}$ if the source time history is a zero-phase wavelet. ${ }^{17,20,22}$

\section{A. Seismic radio-theory}

In the seismic radio application, the source location is known (at point $\mathbf{s}$ ) and the excitation times are unknown. Here, we are after the excitation times, so that a time-coded series of bits can be received and decoded. For this purpose, Eq. (1) is modified to be

$$
m(t)=\sum_{g} d\left(\mathbf{g}, t \mid \mathbf{s}, t_{\text {source }}\right) * g(\mathbf{s},-t \mid \mathbf{g}, 0),
$$

where $d\left(\mathbf{g}, t \mid \mathbf{s}, t_{\text {source }}\right)$ represents the coded message recorded at locations $\mathbf{g}$ for a source at $\mathbf{s}$ with unknown excitation time $t_{\text {source. }}$. The calibration record $g(\mathbf{s},-t \mathbf{g}, 0)$ accounts for the direct wave but also contains all of the primaries, multiples, and scattered waves. Equation (2) can be interpreted as the cross correlation of the coded message with the reference Green's function associated with the source point. In practice, the recorded Green's function is band-limited in the frequency spectrum.

The coded message can be sent using a 6-bit code, with equal (on) and (off) intervals, a system similar to the Baudot code that was invented in 1870 and modified in 1901 by Donald Murray. The suggested coding system is not similar to Morse code, since Morse code is considered to be a zerostate system. Zero-state systems are systems which have three states, 1,0 , and -1 , in Morse codes corresponding to dash, pause, and dot type messages. The system we suggest for use by a TRM system is a non-zero-state system, where it has only on and off which corresponds to signal and no-signal. In this system, each bit is either a signal and interpreted as "1" or no-signal and interpreted as " 0 "; receiving and decoding a string of ones and zeros will give the corresponding letters and spaces. Six elements will be employed.

(1) Signal is interpreted as " $1 . "$

(2) No-signal is interpreted as " $0 . "$

(3) (111111) for the beginning of the message.

(4) (000000) for spaces between words.

(5) (110110) for end of message.

(6) Each group of 6-successive bits will represent one letter.

Results from Eq. (2) are divided into equal intervals, where each interval represents one bit of the decoded message. If the maximum value of each interval exceeds a preselected threshold value, then that interval is interpreted as " 1 ," otherwise the interval is interpreted as " 0 " [Eq. (3)]. The threshold value can range between 0.55 (Ref. 17) and 0.95

$$
\begin{aligned}
& \text { bit }- \text { value }=1, \max (m(t)) \geq \text { threshold, } \\
& \text { bit }- \text { value }=0, \max (m(t))<\text { threshold, }
\end{aligned}
$$

where bit-value represents the decoded value of the corresponding interval. Each group of successive 6 bits is then interpreted as one letter, and hence the whole message can be decoded. A denser coding strategy can be based on discriminating weak from moderate to strong amplitude levels in the signal.

\section{B. Tracking moving objects-theory}

In this application, a group of receivers is permanently buried in the area of interest. A number of stations are then selected. One reference Green's function is recorded for a source at each station. In practice, a metal plate is placed on the ground at the $\mathbf{s}$ location. Recorded traces are stacked together from repeated hammer blows to the plate. No more stacks are needed if additional stacks do not noticeably change the final stacked trace. Later, the same group of receivers will continuously record any passive seismic activity and send it to the processing station to monitor any abnormal activities in the area of interest.

In tracking moving objects on the ground (e.g., cars or moving humans) both the excitation times and the source locations are unknown. In this case, Eq. (1) is used to find the excitation times and source locations, where $g(\mathbf{x}, t \mid \mathbf{g}, 0)$ represents the previously recorded Green's functions and $d\left(\mathbf{g}, t \mid \mathbf{s}, t_{\text {source }}\right)$ represents the passive seismic data.

Correlation results from Eq. (1) are contoured and rendered as 2D maps as a function of the trial source coordinates $(x, y)$, where each map corresponds to a specified trial excitation time. Local maximums of $|m(\mathbf{x}, t)|$ that exceed a pre-selected threshold pinpoint the location of the moving object. Repeating this process for different trial excitation times gives the location of the moving objects at different times. The velocity of the moving object can be approximately estimated based on this information.

The inline and crossline offsets between the receivers/ shots do not need to be uniform. However, it is important to have a sufficiently dense coverage of both receivers and calibration shots to accurately locate the moving object. The required density can be estimated by computerized experiments, but according to our experiments a good criterion might be a sampling interval of $\lambda / 2$, where $\lambda$ is the dominant wavelength of the propagated seismic waves. Processing time is very fast (requiring about $2 \mathrm{~s}$ on a personal laptop), thus allowing almost real time monitoring of the area of interest, where each 2D map is delayed in real time by about $2 \mathrm{~s}$.

The required number of receivers in a TRM system has been studied in other publications. ${ }^{17,23,24}$ For example, in Ref. 17 the number of receivers increased from 1 to 8 , which did not change the resolution but increased the contrast.

Because the propagation and noise environments vary from one field site to the next, it is always recommended that field tests be first conducted to determine the optimal receiver configuration. Similar to seismic land surveys conducted by oil companies, tests are first carried out using oversampled receiver configurations in order to design the most cost effective acquisition geometry for the production survey. Prior to field testing analytical tools that model the migration Green's function ${ }^{25}$ can be used to assess spatial resolution as a function of acquisition geometry. 
a) Sketch showing data acquisition

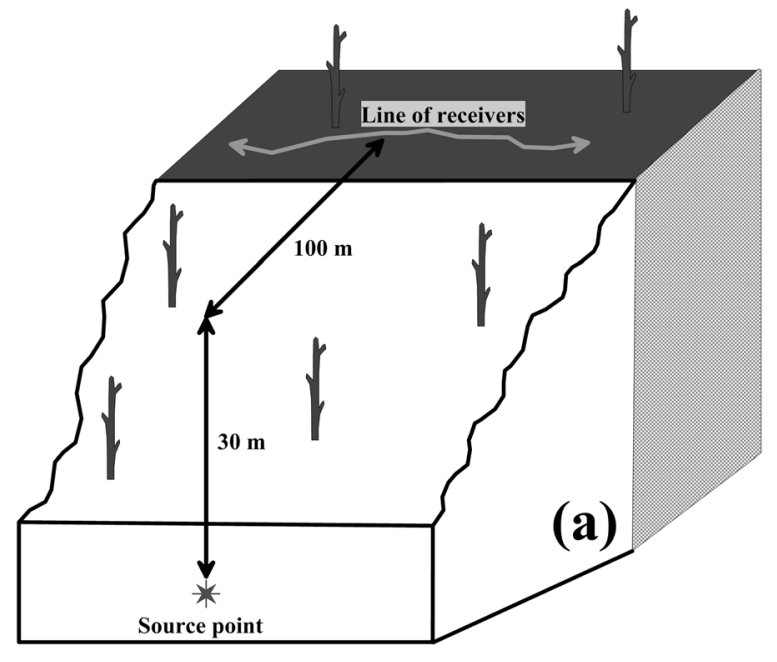

b) A photo taken at the test site

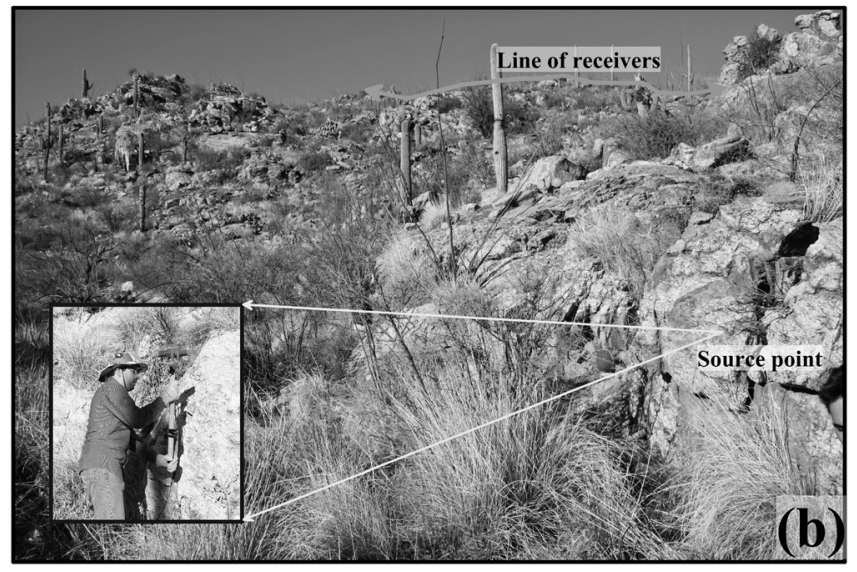

FIG. 1. A photo shows data collection near Tucson, AZ, USA.

\section{FIELD DATA TESTS}

\section{A. TRM seismic radio-numerical tests}

A field test of the TRM seismic radio was carried out in Tucson, AZ (Fig. 1). The selected site is characterized by a high plateau surrounded by a number of valleys, trees, and canyons. A line of 72 receivers running in the $x$ direction is placed at the plateau with a $5 \mathrm{~m}$ receiver interval. The source is located at the offset of $(100,-30) \mathrm{m}$ in the $y$ and $z$ directions [Fig. 1(a)] relative to the center of the receiver line. One shot gather is recorded with 30 stacks [Fig. 2(a)] to be used as the calibration record $g(\mathbf{x}, t / \mathbf{g}, 0)$ in Eq. (2) to decode the message.

Several short messages are fired at the source point and received by the line of receivers [Fig. 2(b)]. The messages are sent using a sledgehammer that directly strikes the rocks. The recorded messages are correlated with the reference shot gather and the results are shown in Fig. 3(a). Here, we did not start the message with (111111) or end it with (110110) to save recording time.

To test the effect of the background noise on the results, random noise is synthetically generated and then band-pass filtered to have the same frequency content as the field data. The synthetic noise is added to the message recorded at the field site and Fig. 2(c) shows the result, where the SNR is lowered by a factor of 340 times. The noisy message is decoded using the reference shot gather and the result is shown in Fig. 3(b). The coded message can be accurately decoded, even after lowering the SNR by 340 times. This is due to the super stacking property of the TRM (for more details on super stacking property see Ref. 20). Scattered events can roughly increase the spatial resolution by a factor proportional to $\sqrt{N M}$, where $N$ is the number of traces and $M$ is the number of events in the data.
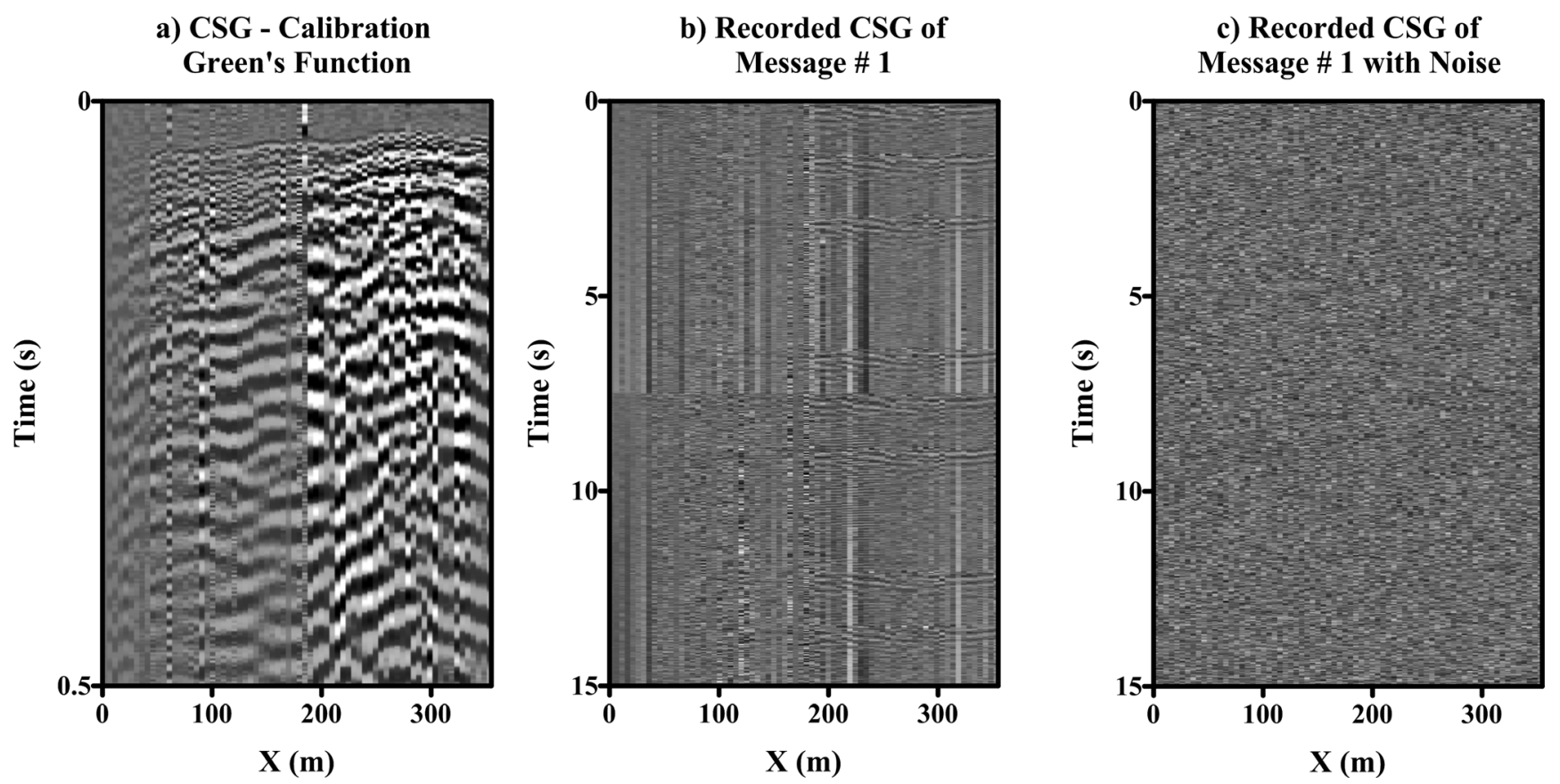

FIG. 2. (a) The high signal-to-noise ratio shot gather used to decode the coded message, (b) part of the coded message as recorded in the field, and (c) same coded message after adding random noise and decreasing the signal-to-noise ratio by a factor of 340 . 
a) Decoded Message from Raw Data (18 bits)

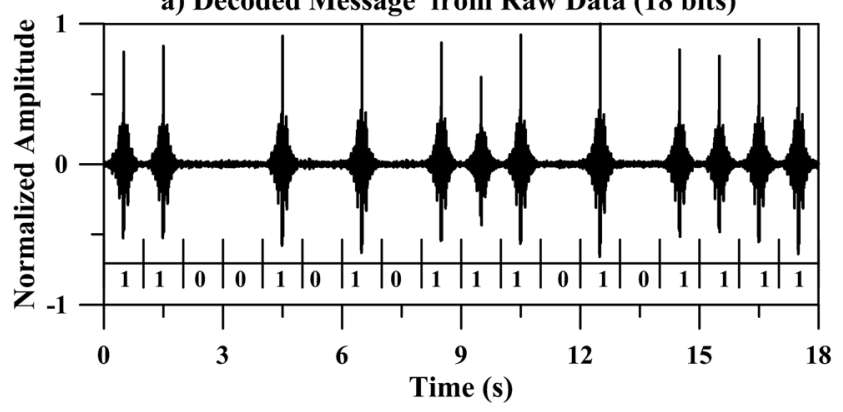

b) Decoded Message from Raw Data + Noises (18 bits)
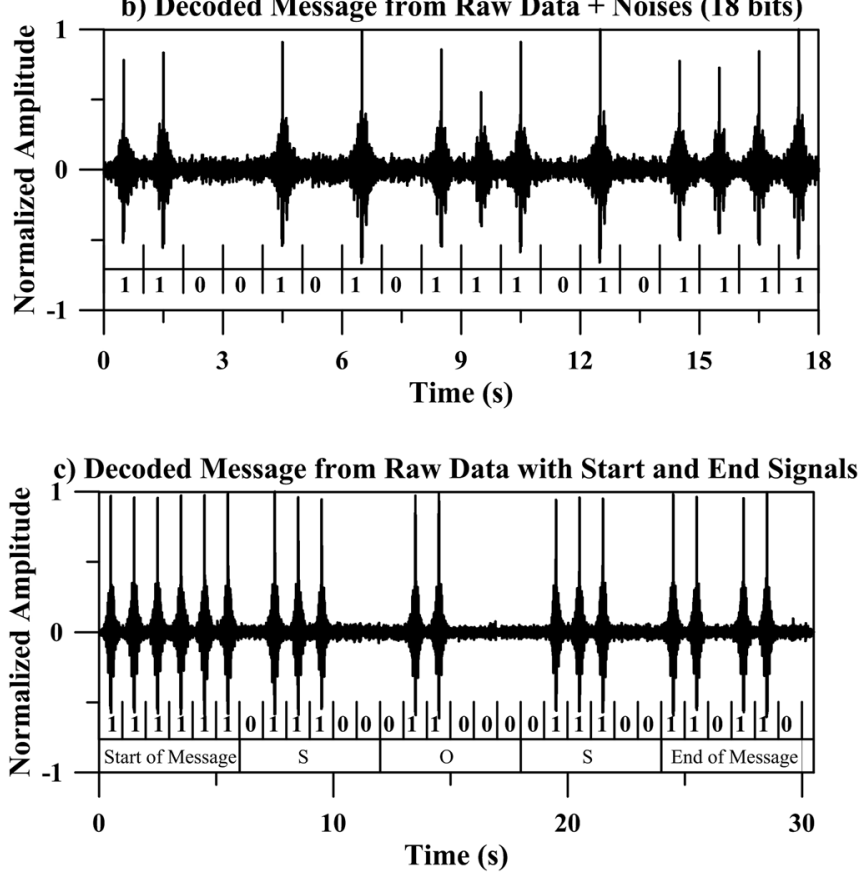

FIG. 3. The decoded messages, where (a) the raw message, (b) the same message as (a) after adding random noises, and (c) another message contains the (111111) at the beginning and (110110) at the end. Message says (SOS), which is an abbreviation for "save our souls."

Another test is conducted where we recorded a message containing the letters (SOS). We designed a key table that shows the letters and the corresponding 6-bit code, but it is not included in this work. In this message [Fig. 3(c)] we started with (111111) and ended with (110110). Results show that the message can be accurately decoded. Here, the time required to send this three-letter message is $30 \mathrm{~s}$, and a few more seconds are required to decode the message. As stated previously the message transmission rate can be theoretically increased by discriminating weak from moderate to strong amplitude levels in the signal.

\section{B. TRM tracking of moving objects-numerical tests}

The application of tracking moving objects is tested with field data. The seismic data are collected at Tucson, AZ, where five parallel receiver lines are used with $5 \mathrm{~m}$ receiver intervals and $15 \mathrm{~m}$ line interval (Fig. 4). Each line contains 24 receiver points, and a reference Green's function is recorded at each receiver point [Fig. 5(a)] by repeatedly
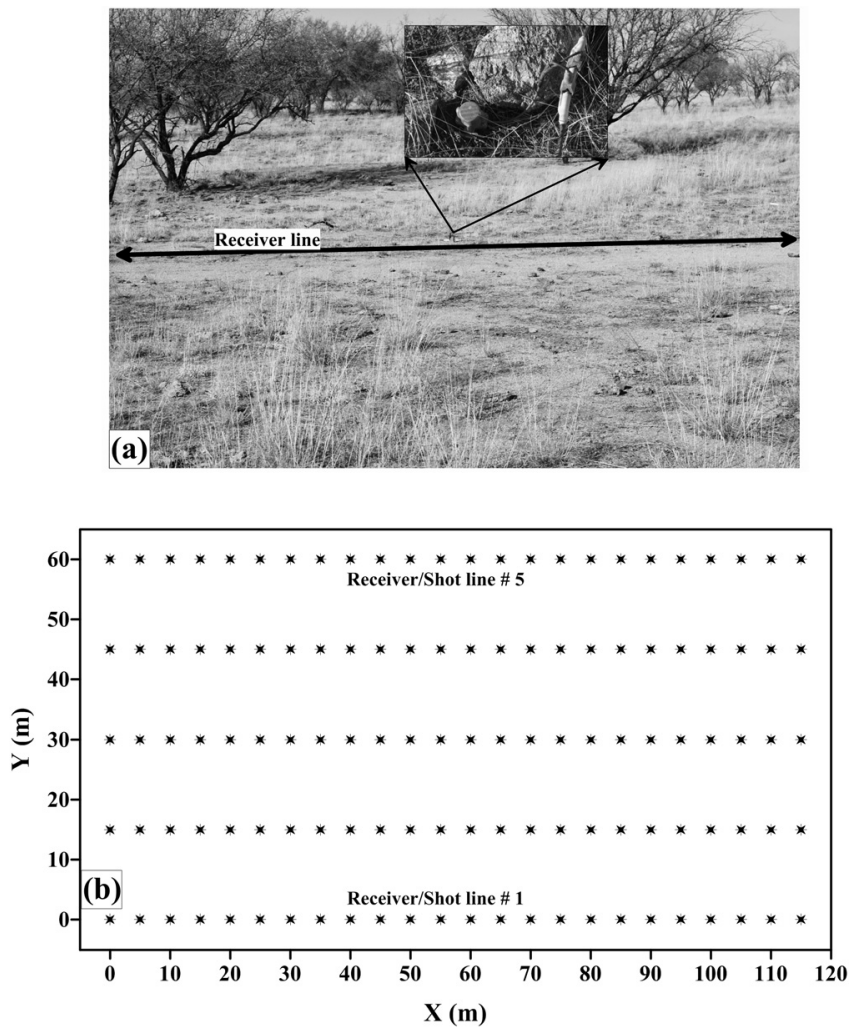

FIG. 4. (a) A photo taken at the test site. (b) A base map shows the locations of the receivers and shot points, arranged in 5 parallel lines, each line contains 24 points. The inline offset is $5 \mathrm{~m}$ and the crossline offset is $15 \mathrm{~m}$. Only receivers were active during the tracking tests, while both sources and receivers were active during the calibration tests.

hammering a metal plate on the ground and stacking the resulting traces recorded by the same geophone. These bandlimited Green's functions are used in Eq. (1) to track moving objects in the area of study. A person moved in the study area following different routes (Fig. 6). He was walking in the first one [Fig. 6(a)] and running in the second [Fig. 6(b)]. We continuously recorded the seismic signals [Fig. 5(b)] during his movement and used the previously recorded Green's functions to track the moving person inside the study area.

Figures 7 and 8 show some snapshots from the correlation results for routes 2 and 3 , respectively. The snapshots are selected so that they show the walker/runner when he is next to one of the geophones. Figures 7 and 8 show that we can accurately track the location of the moving object in the area.

To find the velocity of the moving object we selected part of the path where the object is moving in a straight line. The velocity, $v$, of the moving object is determined by $v=r / t$, where $r$ is the distance between two points along the object track and $t$ is the time required to move between these two points. We calculated two velocity values for each example (walking and running) and the results are

(1) Walking route: the distance between stations 2 and 11, Figs. 7(a) and 7(b), respectively, is $45 \mathrm{~m}$ and the time taken by the walker to move between these two stations is $17.7 \mathrm{~s}$, which gives a velocity of approximately $2.5 \mathrm{~m} / \mathrm{s}$. 


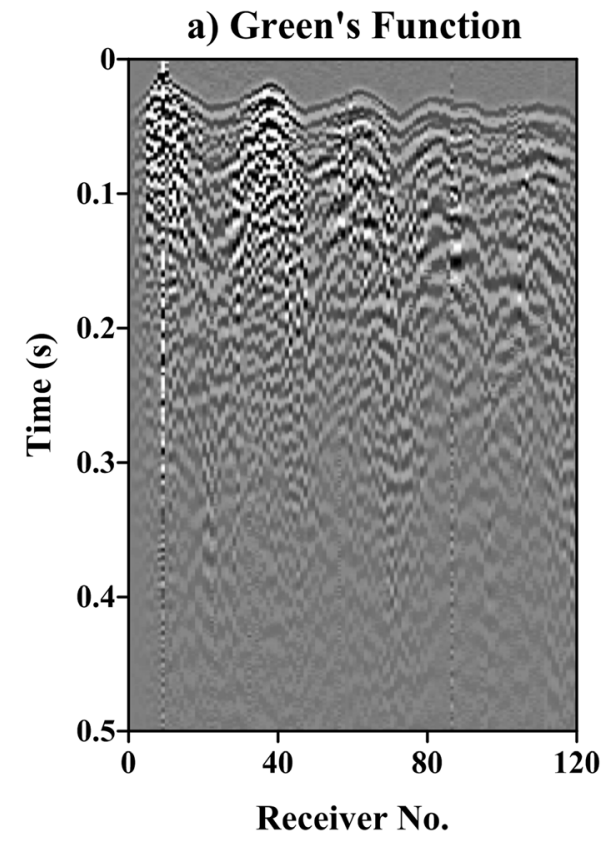

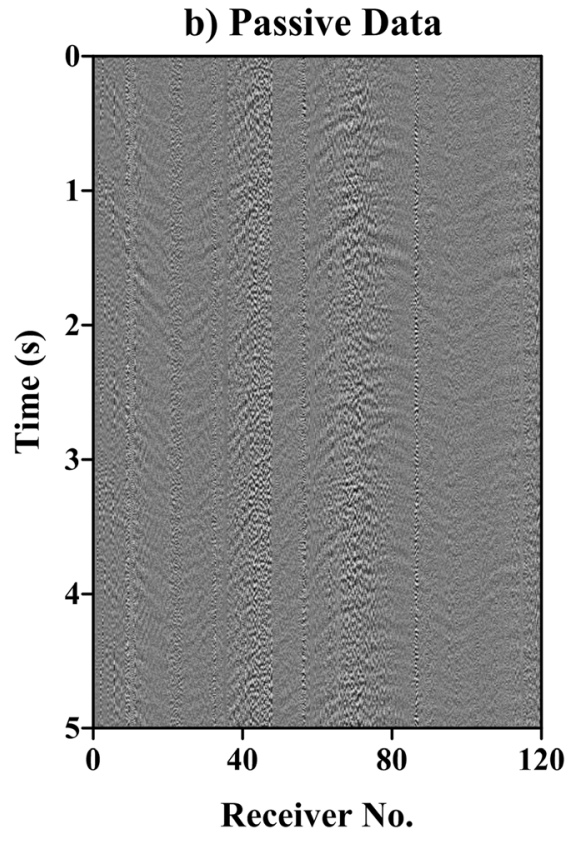

FIG. 5. (a) Green's function for a shot at station 10 and (b) data recorded for a walking person. The dominant wavelength of this data set is calculated to be $13.75 \mathrm{~m}$ where the minimum velocity is $550 \mathrm{~m} / \mathrm{s}$ and the peak frequency is $40 \mathrm{~Hz}$.
(2) Walking route: the distance between stations 108 and 98, Figs. $7(\mathrm{~g})$ and $7(\mathrm{~h})$, respectively, is $50 \mathrm{~m}$ and the time taken by the walker to move between these two stations is $17.5 \mathrm{~s}$, which gives a velocity of approximately $2.9 \mathrm{~m} / \mathrm{s}$.

a) Walking Route

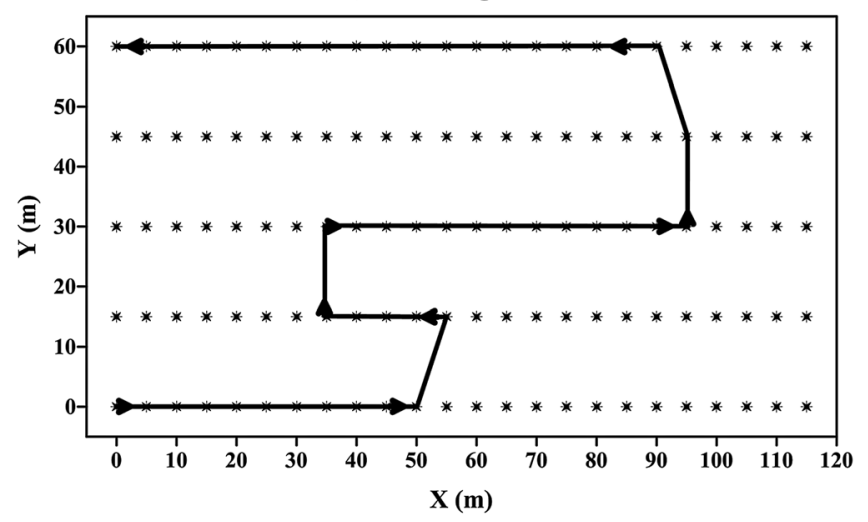

b) Running Route

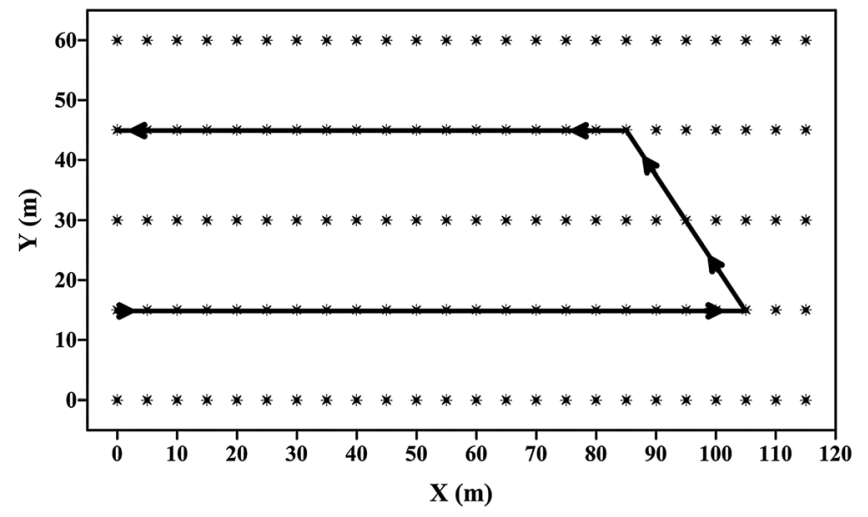

FIG. 6. Sketches show the two different routes taken at the site, where the moving person was walking at (a) and running at (b).
(3) Running route: the distance between stations 25 and 46, Figs. 8(a) and 8(d), respectively, is $105 \mathrm{~m}$ and the time taken by the runner to move between these two stations is $21.1 \mathrm{~s}$, which gives a velocity of approximately $5 \mathrm{~m} / \mathrm{s}$.

(4) Running route: the distance between stations 87 and 74, Figs. 8(f) and 8(h), respectively, is $65 \mathrm{~m}$ and the time taken by the runner to move between these two stations is $11.9 \mathrm{~s}$, which gives a velocity of approximately $5.5 \mathrm{~m} / \mathrm{s}$.

\section{Accuracy test}

It is important to know how accurate the tracking-moving-object technique is, i.e., what should be the distance between the moving object and the receiver/shot point to accurately track it? To answer this question, we selected the snapshot at time $=48.6 \mathrm{~s}$ from route 2 , when the moving object was next to station 61 [Fig. 9(a)] and the five snapshots in Figs. 9(b)-9(f), where the time interval between each of the two snapshots is 0.45 s. In Figs. 9(b)-9(f) all amplitudes are normalized with respect to the values in Fig. 9(a). In Fig. 9(a), the maximum amplitude is exactly at station 61, in Fig. 9(b), which is $0.45 \mathrm{~s}$ later and the walking person is around $1 \mathrm{~m}$ away from station 61, the maximum amplitude value in that snapshot is still at station 61 , but its value is less than that at Fig. 9(a). Moving away from station 61 will gradually decrease the correlation amplitude value, until we are close enough to station 62, where a new peak is formed.

This test shows that, the maximum correlation value occurs when the moving object is exactly at receiver/shot location, moving away from that location decreases the correlation value. If the distance between receiver/shot points is too large, we may not be able to accurately locate the moving object. This problem can be eliminated by using a finer sampling of source points when creating the calibration Green's functions. The optimum offset between receiver/ shot point depends on the site condition, sampling interval of 
(a) Snapshot @ time $=3.5 \mathrm{~s}$

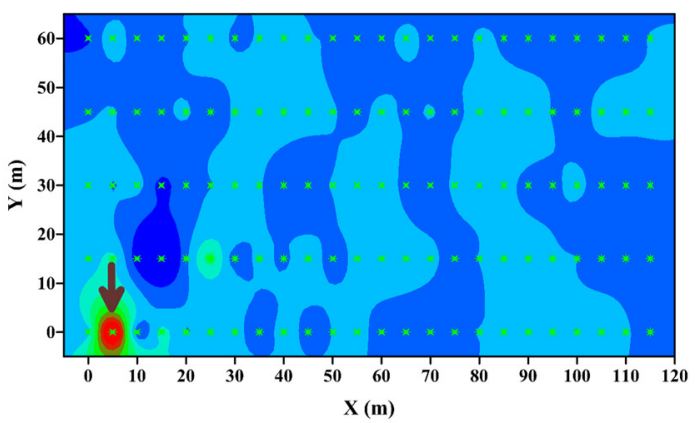

(c) Snapshot @ time=30.6 s

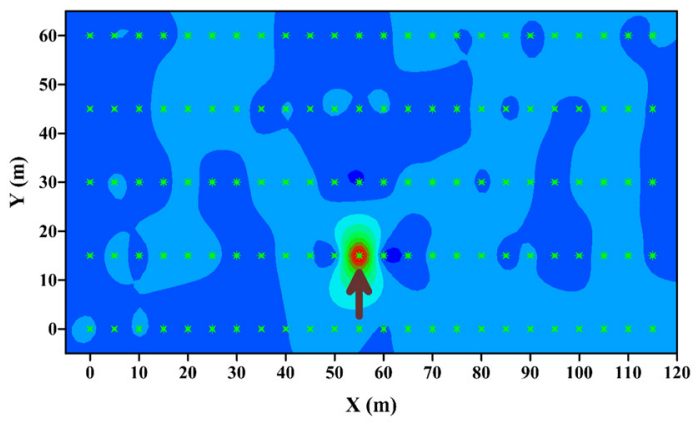

(e) Snapshot @ time=61.9 s

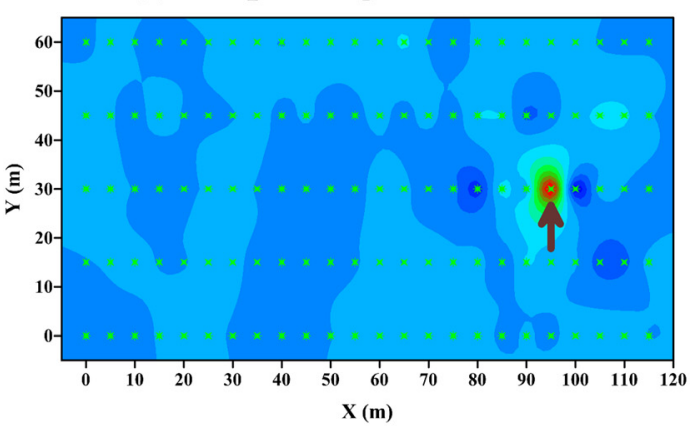

(g) Snapshot @ time=94.7 s

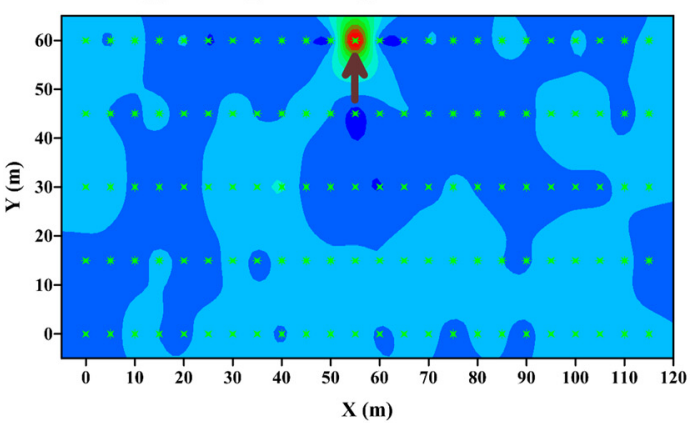

(b) Snapshot @ time = 21.2 s

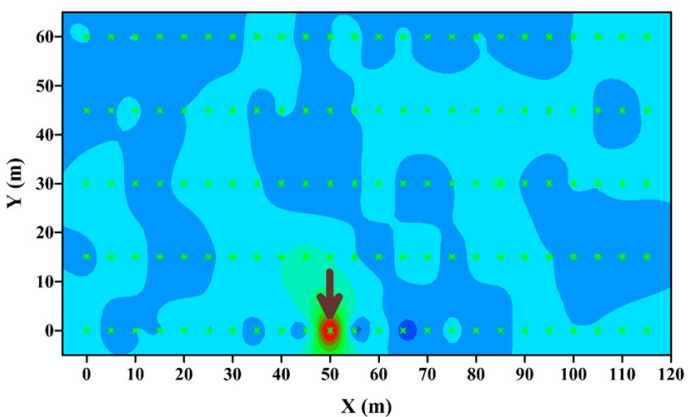

(d) Snapshot @ time=48.6 s

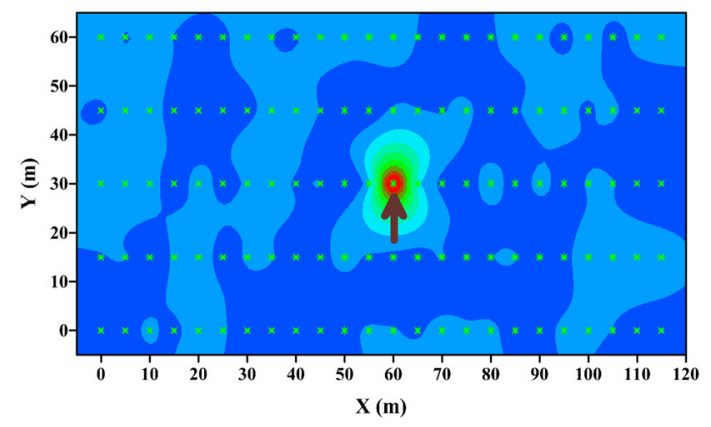

(f) Snapshot @ time=80.9 s

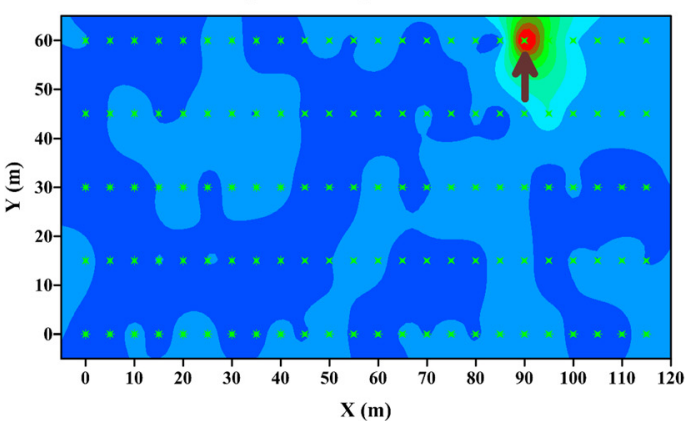

(h) Snapshot @ time=112.2 s

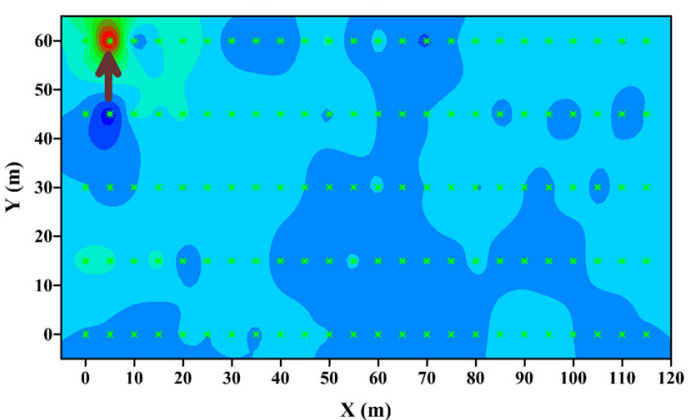

Normalized correlation amplitude

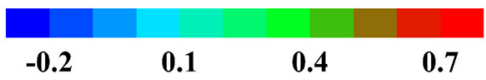

FIG. 7. (Color online) Snapshots at different times show the correlation results for a walking person. Location of the walking person is characterized by high correlation values. Arrow refers to the actual location of the walking person.

the calibration Green's functions, background noise, and SNR of the reference Green's function. The frequency response of the receivers could be a minor factor in determining accuracy, but we did not test this in the current work.

\section{SPATIAL RESOLUTION ESTIMATE}

The spatial resolution of the TRM mirror is proportional to that for zero-offset migration. ${ }^{26}$ This means that the Rayleigh resolution formula is appropriate for estimating the 
(a) Snapshot @ time =1.8 s

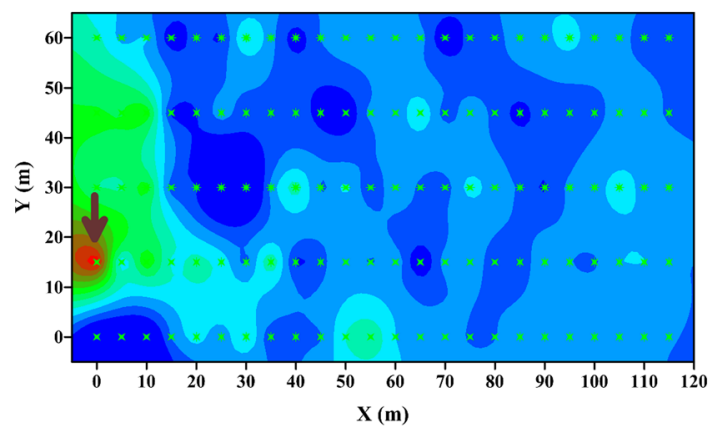

(c) Snapshot@ time=14.5 s

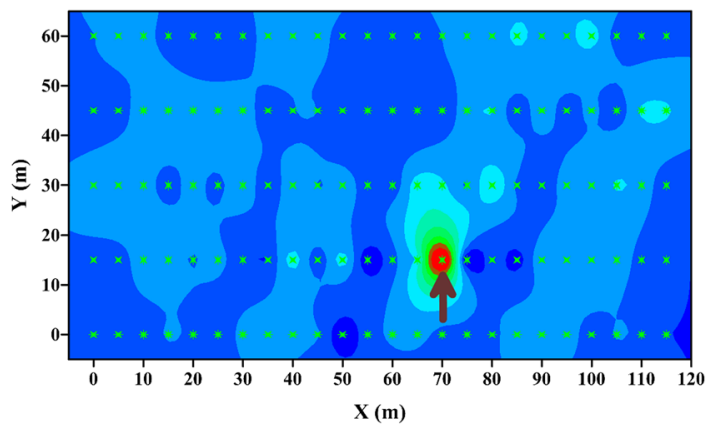

(e) Snapshot @ time = 40.7 s

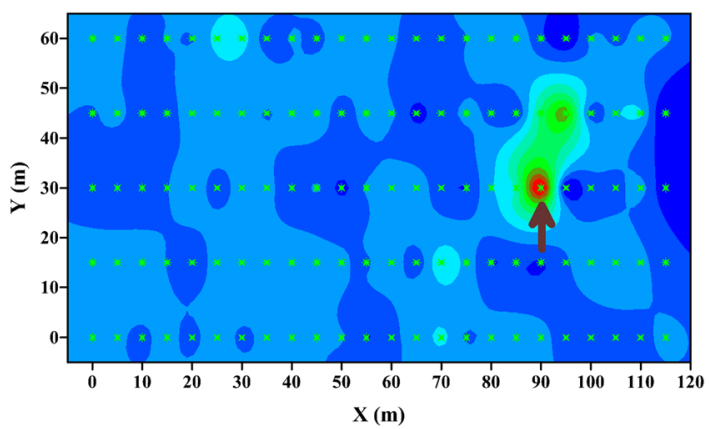

(g) Snapshot@ time= $54.2 \mathrm{~s}$

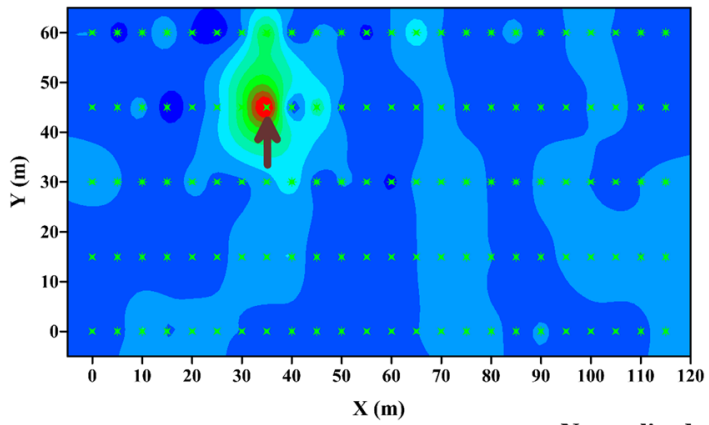

(b) Snapshot @ time=5.5 s

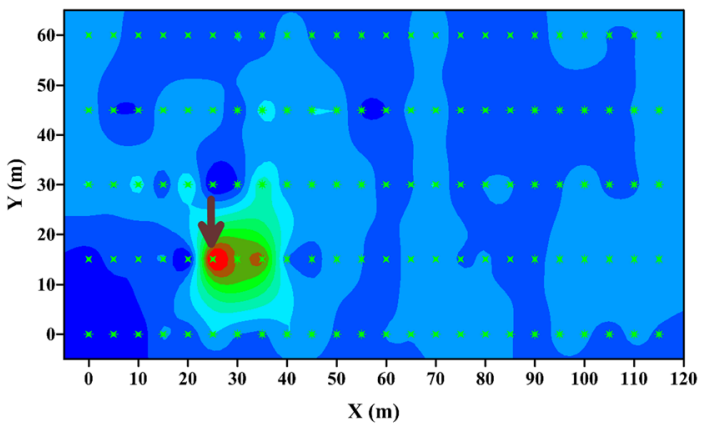

(d) Snapshot@ time=22.9 s

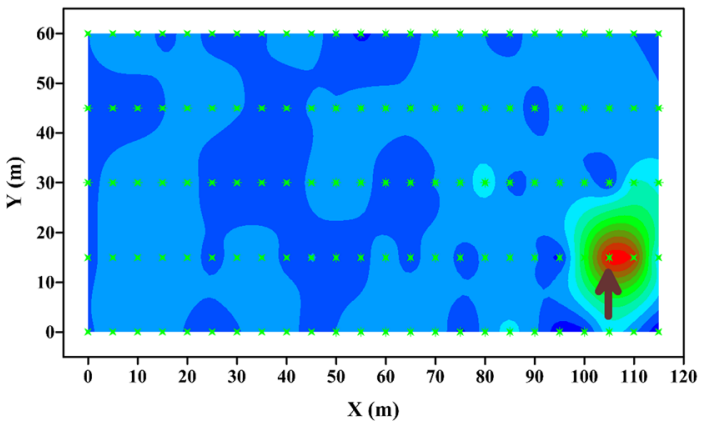

(f) Snapshot @ time = 48.3 s

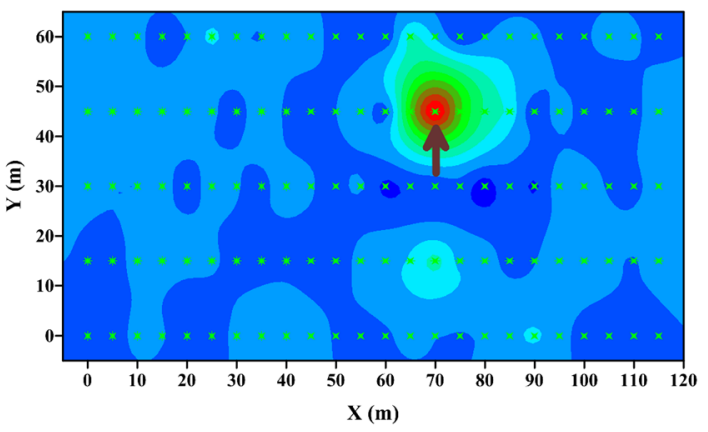

(h) Snapshot @ time=60.2 s

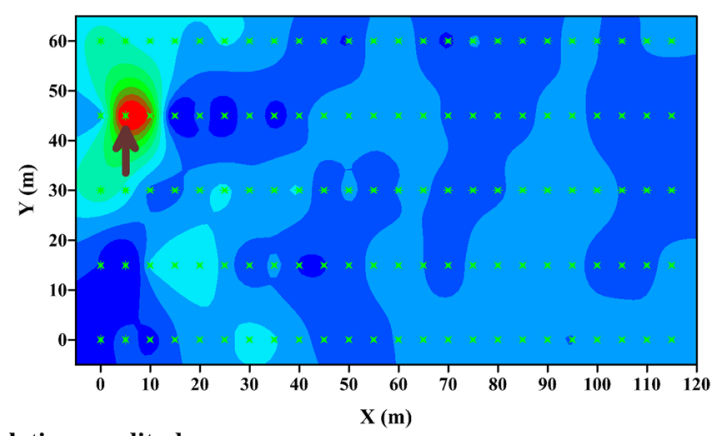

$0.5 \quad 0.9$

FIG. 8. (Color online) Snapshots at different times show the correlation results for a running person. Location of the running person is characterized by high correlation values. Arrow refers to the actual location of the running person.

spatial resolution limits of the TRM image. If $\Delta x$ is defined as the lateral distance between two point scatterers that can just be distinguished in the TRM image, then in the farfield approximation

$$
\Delta x=\frac{\lambda Z}{4 L},
$$

where $\lambda$ is the dominant wavelength of the interfering waves, $Z$ is the shortest distance between the point scatterer and a 
(a) Snapshot @ time=48.6 s

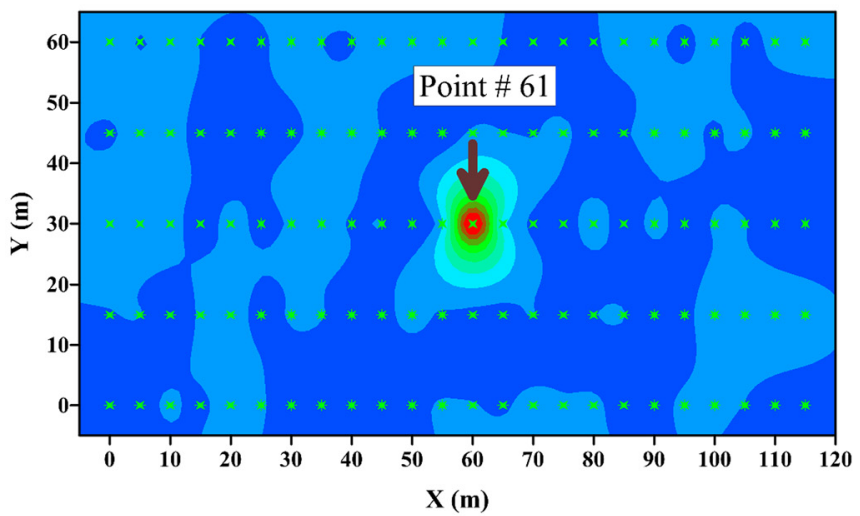

(c) Snapshot @ time=49.5 s

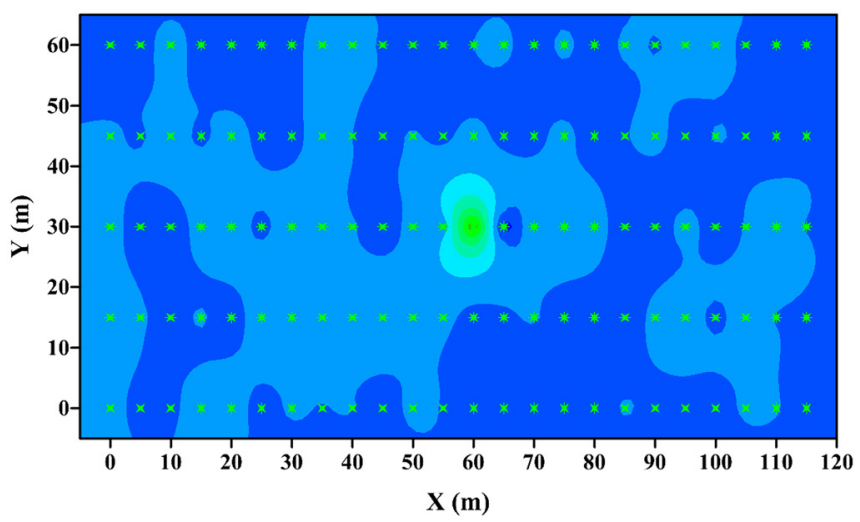

(e) Snapshot@ time=50.4 s

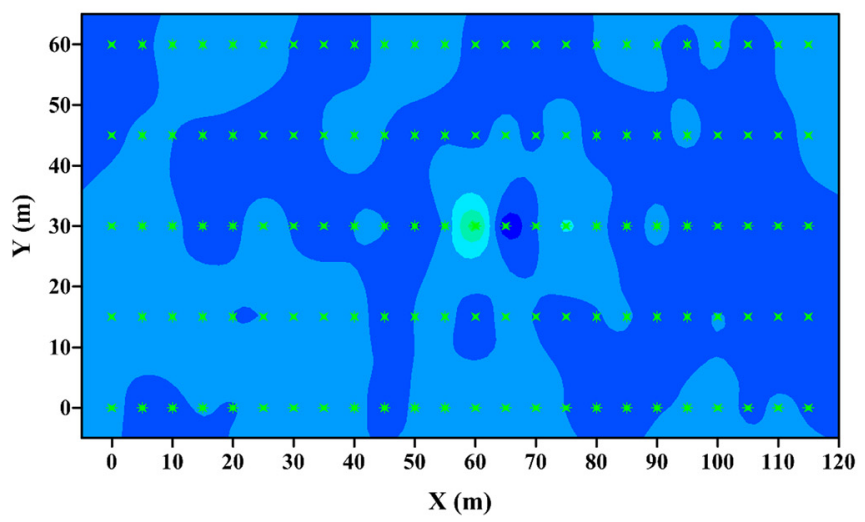

(b) Snapshot @ time $=49.0 \mathrm{~s}$

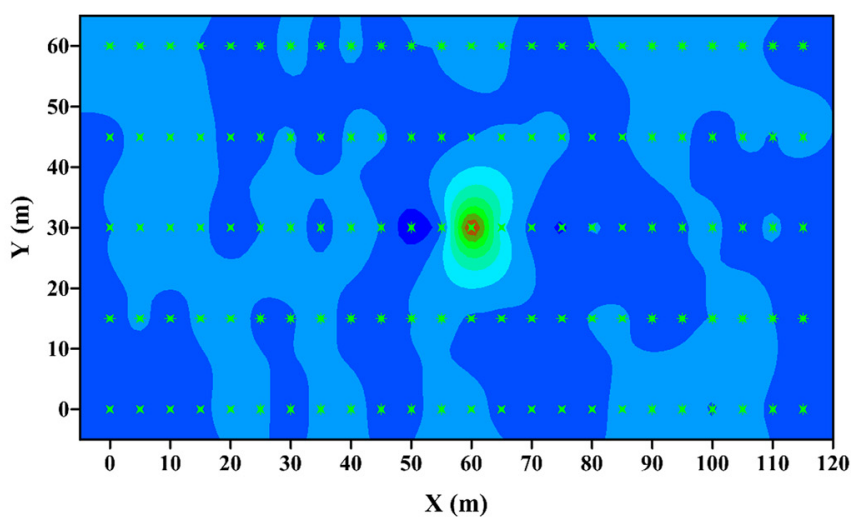

(d) Snapshot @ time $=49.9 \mathrm{~s}$

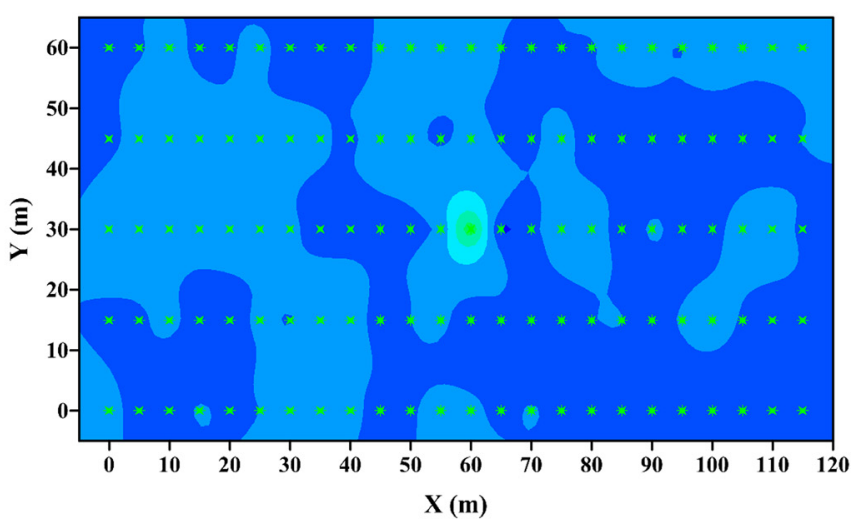

(f) Snapshot@ time=50.9 s

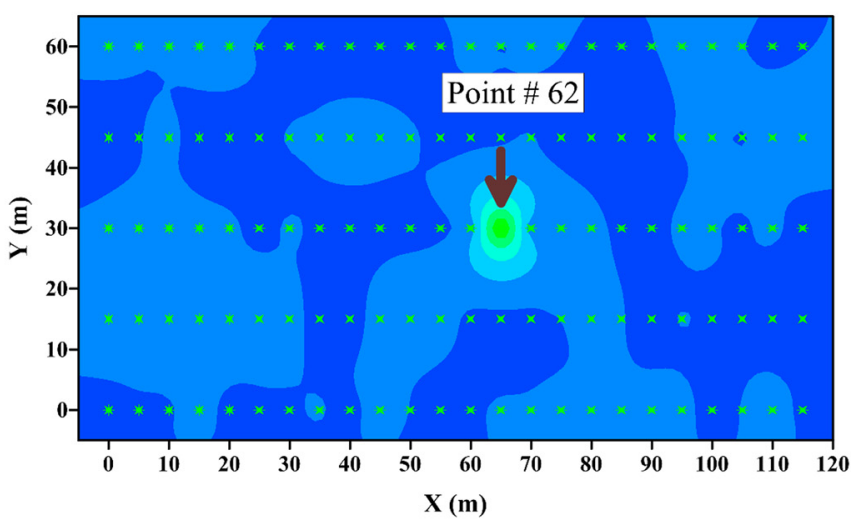

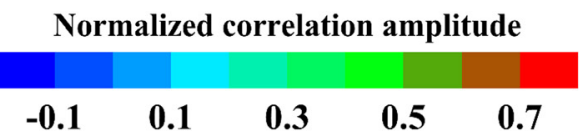

FIG. 9. (Color online) Snapshots show the correlation result when the moving object is: (a) at station 61 , and (b)-(f) the following five shots with $0.45 \mathrm{~s}$ time intervals between each.

lateral line of receivers, and $L$ is the length of the straight receiver line. This formula is valid for primary transmission events, but if multiple scattering is significant then the resolution can approach that of the Abbe limit $\lambda / 2$. We conjecture that most natural environments are rich in strongly scattered multiples. An analytical formula for determining zero-offset resolution and the strength of side lobe artifacts for different receiver configurations is given in Ref. 27.

\section{DISCUSSION ON LIMITATIONS OF THE METHOD}

A reference Green's function with a low SNR could decrease the accuracy of the results in both the radio and radar applications. The reference Green's functions are used as calibration records to decode the messages or track the moving objects, and empirical evidence suggests that recording them with SNR $>4$ is likely to produce robust detection of signals. In the seismic radio application it is important to 
keep fixed bit time-intervals, otherwise the message will not be accurately decoded. Thus, a well-trained person should send the message or an automated source should be used. In the seismic radar application, it is important to have a sufficiently dense coverage of reference shot gathers to accurately locate the moving object; our field experiments showed that $\Delta x_{s} \leq \lambda / 2$ will give accurate results, where $\Delta x_{s}$ is the offset between shot points in the $x$ and $y$ directions.

There are other factors which have little influence on the results such as the receiver alignment or interval, or the climatic conditions (temperature, humidity, etc.). Temperature changes in the air will not significantly affect the speed of seismic waves propagating in the ground. On the other hand, the level of noise may affect the accuracy of the results. Our test showed that lowering the SNR by a factor of 340 has a minor effect on the results. We emphasis that only random white noise was added to the recorded data, and did not test the case for coherent noise.

Another factor that can degrade the effectiveness of a TRM system is the change in the subsurface conditions due to ground collapse or soil sliding. Small to moderate subsurface changes will not dramatically change the reference shot gathers, ${ }^{28}$ and hence the reference shot gathers will still be valid. However, a major change in the topographic landscape on the scale of a wavelength will likely require updating the reference shot gathers.

\section{CONCLUSIONS}

Two seismic applications of the TRM method are introduced. In both applications a group of reference records is required by initially recording band-limited Green's functions with SNRs better than about 4:1.

The first application is receiving and decoding coded messages using seismic TRMs. The message can be coded using 6-bits per letter scheme, where the beginning and ending of the message should be unique. The recorded message is correlated with the previously recorded Green's function and the results are interpreted as " 1 " or " 0 " depending on the correlation value. This application can be used to receive coded messages from trapped miners where no other means of communication, such as radio waves, is possible. A denser coding strategy can be based on discriminating weak from moderate to strong amplitude levels in the signal. Another possible application is communicating geologic and log information at the drill bit to the surface during drilling operations.

The second application is tracking moving objects by TRM radar. A group of receivers are buried in the area of interest and continuously record the passive seismic data from any moving object in that area. The passive data are correlated with the previously recorded Green's function and the high amplitude value refers to the location of the moving object. Tracking of the moving objects can be done in almost real time and the approximate velocity of that object can be easily calculated.

Two field tests were carried out in Tucson, AZ to test the proposed TRM applications. Results show that coded messages can be accurately decoded and the location and ve- locity of moving objects can be accurately estimated. This assumes that on and off signals have nearly equal intervals in the first application, and the shots are densely located in the second.

Future work in these methods includes another field test conducted in a working mine to test the dense coding strategy, where amplitudes are used as independent information in the coded message.

\section{ACKNOWLEDGMENTS}

We would like to thank the 2009/2010 sponsors of the CSIM Consortium for their support. We would like to thank Paul Gettings and Ernesto Curiel for all the help they provided during data acquisition. Trans. Ultrason. Ferroelectr. Freq. Control 39, 555-566 (1992).

${ }^{2}$ M. Fink, "Time-reversal mirrors," J. Phys. D 26, 1333-1360 (1993).

${ }^{3}$ A. Parvulescu and C. S. Clay, "Reproducibility of signal transmission in the ocean," Radio Electr. Eng. 29, 223-228 (1965).

${ }^{4}$ W. S. French, "Two-dimensional and three-dimensional migration of model-experiment reflection profiles," Geophysics 39, 265-277 (1974).

${ }^{5}$ G. A. McMechan, "Migration by extrapolation of time dependant boundary values," Geophys. Prospect. 31, 413-420 (1983).

${ }^{6}$ N. D. Whitmore, "Iterative depth migration by backward time propagation," Expanded Abstracts of SEG, 382-385 (1983).

${ }^{7}$ W. Chang and G. A. McMechan, "Reverse-time migration of offset vertical seismic profiling data using the excitation-time imaging condition," Geophysics 51, 67-84 (1986).

${ }^{8}$ W. F. Chang and G. A. McMechan, "3-d acoustic prestack reverse-time migration,” Geophys. Prospct. 38, 737-775 (1990).

${ }^{9}$ R. Sun and G. A. McMechan, "Scaler reverse-time depth migration of prestack elastic seismic data," Geophysics 66, 1519-1527 (2001).

${ }^{10}$ B. E. Anderson, M. Griffa, P. L. Bas, T. J. Ulrich, and P. A. Johnson, "Experimental implementation of reverse time migration for nondestructive evaluation applications," J. Acoust. Soc. Am. 129, EL8-EL14 (2011).

${ }^{11}$ A. Parvulescu, "Matched-signal ("mess") processing by the ocean," J. Acoust. Soc. Am. 98, 943-960 (1995).

${ }^{12}$ W. A. Kuperman, W. S. Hodgkiss, H. C. Song, T. Akal, C. Ferla, and D. R. Jackson, "Phase conjungation in the ocean: experimental demonstration of an acoustic time- reversal-mirror," J. Acoust. Soc. Am. 103, 25-40 (1998).

${ }^{13}$ H. C. Song, W. A. Kuperman, and W. S. Hodgkiss, "A time-reversal mirror with variable range focusing," J. Acoust. Soc. Am. 103, 3234-3240 (1998).

${ }^{14}$ G. F. Edelmann, H. C. Song, S. Kim, W. S. Hodgkiss, W. A. Kuperman, and T. Akal, "Underwater acoustic communications using time reversal," IEEE J. Oceanic Eng. 30, 852-864 (2005).

${ }^{15}$ J. de Rosny and M. Fink, "Overcoming the diffraction limit in wave physics using a time-reversal mirror and a novel acoustic sink," Phys. Rev. Lett. 89, 124301 (2002).

${ }^{16}$ A. Derode, A. Tourin, J. de Rosny, M. Tanter, S. Ton, and M. Fink, "Taking advantage of multiple scattering to communicate with timereversal antennas," Phys. Rev. Lett. 90, 014301 (2003).

${ }^{17}$ R. K. Ing and N. Quieffin, "In soild localization of finger impacts using acoustic time-reversal process,” Appl. Phys. Lett. 87, 204104 (2005).

${ }^{18}$ G. Lerosey, J. de Rosny, A. Tourin, and M. Fink, "Focusing beyond the diffraction limit with far-field time reversal," Science 315, 1120-1122 (2007).

${ }^{19}$ S. Gaffet, T. Monfret, G. Senechal, D. Rousset, and H. Zeyen, "Seismic time reversal experiment in the low noise underground laboratory, LSBB (France)," Geophys. Res. Abstr. 7, SRef-ID: 1607-7962/gra/EGU05-A08256 (2005).

${ }^{20}$ S. Hanafy, W. Cao, and G. Schuster, "Using super-stacking and superresolution properties of time-reversal mirrors to locate trapped miners," TLE 28, 302-307 (2009).

${ }^{21}$ I. Lokmer, G. S. O'Brien, D. Stich, and C. J. Bean, “Time reversal imaging of synthetic volcanic tremor sources," Geophys. Res. Lett. 36, L12308 (2009). 
${ }^{22}$ S. Kaartvedt, A. Rostad, T. A. Klevjer, and A. Staby, "Use of bottommounted echo sounders in exploring behavior of mesopelagic fishes," Marine Ecol. Prog. Ser. 395, 109-118 (2009).

${ }^{23}$ A. Derode, A. Tourin, and M. Fink, "Ultrasonic pulse compression with one-bit time reversal through multiple scattering," J. Appl. Phys. 85, 6343-6352 (1999).

${ }^{24}$ S. Yon, M. Tanter, and M. Fink, "Sound focusing in rooms: II. The spatio-temporal inverse filter," J. Acoust. Soc. Am. 114, 3044-3052 (2003).
${ }^{25}$ G. T. Schuster and J. Hu, "Greens function for migration: Continuous recording geometry," Geophysics $\mathbf{6 5}, 167-175$ (2000).

${ }^{26} \mathrm{G}$. T. Schuster, Seismic Interferometry (Cambridge University Press, New York, 2009), pp. 211-228.

${ }^{27}$ J. Chen and G. T. Schuster, "Resolution limits of migrated images," Geophysics 64, 1046-1053 (1999).

${ }^{28}$ W. Cao, S. M. Hanafy, G. T. Schuster, G. Zhan, and C. Boonyasiriwat, "High-resolution and super-stacking of time reversal mirrors in locating seismic sources," Geophys. Prospect, doi: 10.1111/j.1365-2478.2011.00957.x. 